\title{
A MANAGEMENT APPROACH TO TOTAL COMPUTER PRODUCTIVITY AT GODDARD SPACE FLIGHT CENTER
}

\author{
Evmenios P. Damon
}

\author{
Mission Operations Computing Division \\ Goddard Space Flight Center \\ Greenbelt, Maryland 20771
}

\begin{abstract}
Summary
The management of the Mission Operations Computing Facility at the Goddard Space Flight Center established a plan which assured total computer productivity. Furthermore, the plan safeguarded against unwarranted expansion of the Facility consisting of four large-scale digital computers. The elements of the plan are: (a) optimization of the facility, (b) optimization of the present workload, (c) optimization of the new workload, and (d) establishment of continuous communication channels with the users. Throughout the discussion of the plan, it becomes evident that when management provides leadership and operating managers actively and enthusiastically cooperate with professional computer staffs, major economic achievements can result.
\end{abstract}

\section{Introduction}

Goddard Space Flight Center's (GSFC's) computer experience began with the use of two IBM 704's during Project Vanguard in the mid-50's. As the Space Program was growing in scope and complexity and spacecraft technology was advancing at a rapid pace, GSFC's computer requirements were also growing at a very high rate.

In 1966 the first 360, a Model 65 computer, came to GSFC. Within the following two years, the Missions Operations Computing Facility (MOCF) acquired, in addition to the $360 / 65$, two 360/75's and a 360/95. This facility collectively supports, around-the-clock, manned and unmanned spacecraft orbit computations, unmanned attitude determination and control, manned spacecraft tracking data analysis, spacecraft command and control, satellite tracking and data network computations, and research and development in all phases of aerospace technology.

The MOCF is operated and managed by the Mission and Data Operations Directorate through the Mission Operations Computing Division (MOCD).

\section{A Management Challenge}

By mid-1969 this powerful computing facility was brought down to its knees by the increased demand in services by the user community. It appeared to management and users alike that the sum of all the demands on the facility plus the "overhead" of allocating, scheduling, and mediating the services demanded exceeded the capacity of the facility. The users no longer were able to interact as intensively as they were used to, with what had previously appeared to each one of them to be their own large and capable computers.

After the initial shock, management spurred into immediate action. A management approach which would solve the immediate problem of computer "saturation," assure total productivity, and guarantee against unwarranted expansion was established. The management plan called for (a) optimization of the facility, (b) optimization of the present workload, (c) optimization of the new workload, and (d) establishment of communication channels with the users. Each one of these will be discussed at some length.

As can be seen from the discussion which follows, when management provides leadership and operating managers actively and enthusiastically cooperate with professional computer staffs, major economic achievements can result.

\section{Facility Optimization}

Management recognized that the primary resources of the computing facility are computer time, computer storage, and computer equipment. Management plans were drawn and implemented in a way that computer time is regulated by preparation of schedules on a weekly basis for each of the computers. Maximum time limits for daytime work and memory requirements have been imposed along with priority treatment for extremely short jobs and special overnight requirements. Computer storage is monitored continuously, particularly the on-line disk storage. Efforts to maintain an active data base serving the primary needs of the Center resolve into registration of users, approval of requests for space, and monitoring the use of disk storage previously granted. Computer equipment usage is constantly monitored in order to maintain an efficient configuration for each computer. Periodic checks are made on the use of remote terminals to insure that the distribution of terminals is in keeping with the workload.

Acquisition of equipment, components and communications facilities, error analyses, exploration of methods of improving computer effectiveness through reconfiguration or interconnection between machines, and interfacing with operational and software personnel in order to achieve optimum performance are an integral part of the management plan.

As new items of equipment are acquired and installed, engineering personnel interface with software and operational personnel to ascertain that the equipment is properly utilized and exploited in the most effective manner. Advance planning is carried out, particularly in conjunction with software personnel, to assure that the operating system is suitably modified to recognize any new piece of equipment to be installed.

In order to maintain continuity of operation for existing applications, compatibility considerations must be taken into account. When new hardware components are acquired, the required degree of compatibility is assured even though the new equipment may be more capable, or less expensive, than that which it replaces.

Devising the best possible arrangement, or configuration, of 
available equipment to meet current requirements is a matter of constant consideration for the MOCD. Reconfiguration to achieve a multiprocessor arrangement may be advisable. Shifting of remote terminal or graphic device support from one machine to another may be required under certain circumstances. Backup capability, particularly during mission support periods, may be necessary for continued operation of daily production work and may demand either temporary or permanent rearrangement of specialized hardware facilities. Close contact and cooperative effort between engineering, software, and operations personnel are maintained.

The current trends which will continue into the Shuttle era are: (a) expansion of remote terminal (job input/output) and computer networking capabilities, (b) job load-leveling and backup via computer interconnection (frontend processors, transmission controllers, channel-to-channel links, and shared disks), and (c) the use of non-IBM (multi-vendor) equipment for cost savings and/or improvements.

It is acutely recognized that all three trends will impose a greater emphasis on data monitoring, control, efficiency, and reliability. Interfaces will have to be (a) fail-safe so that one unit does not impair another, (b) switchable in order to dynamically relocate jobs because of overload or failures, and (c) readily controllable for data manageability and accountability.

From the operating system point of view, it has been the objective of the MOCD to maintain a strong systems programming activity. As purveyors of services, the MOCD does not have an end product toward which progress can be measured. Moreover, the services rendered can be significantly impacted by many activities of the Center, outside the control of the MOCD. These include: (a) the number of NASA missions supported by the Center and the degree and kind of support required, (b) software modifications and augmentations created by suppliers outside GSFC, and (c) contract policies which may modify the type of users served, etc.

However, through the use of software monitors such as the SUPERMON developed by Stanford University, Stanford, Califormia and the System Measurement Software 360 (SMS/360) which is a proprietary product developed by Boole \& Babbage, Inc., Cupertino, California, substantial productivity improvements have been made and continue to be made.

SUPERMON is a Multiprogramming with a Variable Number of Tasks (MVT) software monitor, operating as a system task under OS/360 MVT, designed to monitor hardware and software performance over a given period of time. It produces a series of reports which should aid in identifying areas of low utilization as well as performance bottlenecks. SMS/360 is comprised of two components: the Problem Program Evaluator (PPE) and the Configuration Utilization Evaluator (CUE). Each of these components consists of two parts, an Extractor and an Analyzer.

The PPE Extractor samples the activity of a problem program under investigation. Although the computer may be operating in a multiprogramming mode, the Extractor is active only when that particular program has control of the machine. Hence, the resultant data, written in to a data set called the Extractor data set, gives the appearance that this one problem program is the only one in the machine.

The CUE Extractor collects data on hardware usage, data cell or disk head movement, transient Supervisor Call (SVC) module loading including transient SVC area usage for MVT, device and channel queueing, and initiator activity. This data is output to an Extractor data set, which may be recorded on a magnetic tape or direct access device. The Extractor process continues to sample until the time limit indicated via one of the input pararneters is reached or the stop procedure is performed.

Upon the installation of a new release of the Operating System (OS/360), studies are carried out, and depending on findings, action is taken either to reconfigure the existing enviroament or OS/360 itself.

Several cases in which improvements have been achicved as a result of CUE measurements have been documented and are cited below.

Case 1

There are a series of system modules known as SVC routines which are very frequently used. Many of these routines may be resident in memory or stored on a direct access device at the installation's option. During the time that GSFC had IBM Systems Engineering (S.E.) coverage, they had made a determi iation, based perhaps upon intuition, as to which should be rnemory resident. Subsequent measurements revealed an averaze of 15,000 transfers of SVC routines from drum to memcry per hour, a rather high load on the Input/Output (I/O) charnel, and an excessive delay for the program issuing each SVC. In a test made with no SVC's resident, the figure rose to an averige of 75,000 loads per hour (a measure of total SVC activity). This test indicated which SVC's were used most frequently. Upon making the most heavily used ones resident, the average number of loads dropped to 1,500 per hour, a reduction of 10 to 1 ovirs the former figure. In addition, this arrangement of SVC residency resulted in less memory space being occupied by the system. An additional $50 \mathrm{~K}$ bytes of memory was made available to aser programs $(K=1024)$.

\section{Case 2}

In the Spring of 1971, OS/360 Release 19 was installed on the $360 / 95$. A study was performed again to determine the frequency of accessing SVC's which, as stated above, opiionally may be made resident in the computer memory or on direct access peripheral storage. The study revealed that 16 SVC's residing on peripheral storage accounted for approximately 50 percent of the SVC load. The number of SVC loads had risen from 1,500 per hour (Release 18 ) to approximately 8,800 per hour. Making the above SVC's resident reduced the number of loads to between 1,100 and 1,500 per hour.

\section{Case 3}

During the process of gathering the data cited in Case ? above, additional information also obtained revealed that the CI'U was waiting as much as 58 percent of the time while a particular channel was busy. The devices responsible for most of th: wait time were identified. A determination was made as to system components which would cause arm contention on identified 2314 disk devices and appropriate adjustments were madis. Prior to this adjustment, Central Processing Unit (CPU) utilization was in the neighborhood of 40-73 percent. After system adjistments were made, CPU utilization was observed to be as high as 99 percent.

\section{Case 4}

During June 1971, it was noted that the performance of the $360 / 95$ was below normal. With the exception of some R 6 mote Job Entry (RJE) problems that persisted, the major Release 19 bugs which might have had a bearing on performance had been corrected. Also, the list of resident SVC routines had been revised for the new release (See Case 2). Nonetheless, CUF; confirmed the fact that overall performance was off, with CPIJ 
"busy" figures in the range of 56 percent, after peaking at $90+$ percent.

Thorough analysis of the CUE data indicated the difficulty seemed to be centered about a channel contention problem, and the OS $/ 360$ job queue was centrally involved.

A rearrangement of disk packs was developed and implemented. The new arrangement placed the job queue on Channel 3 , which it shared with only low-usage packs. Scratch packs were spread out over Channels 2 and 5 . In addition, more scratch packs were made eligible to receive SYSOUT data sets.

These changes were placed into effect on June 24, 1971. Performance of the machine improved immediately as shown by CUE measurements, direct observation, and job count.

Because of its ability to insert itself directly into the flow of system events, SUPERMON is able to determine accurately the number and source of frequently-occurring short-lived events such as $\mathrm{I} / \mathrm{O}$ interrupts and system software module usage, and thus present a more valid report than that produced merely by sampling techniques.

\section{I/O Interrupts}

In an effort to reduce the number of $I / O$ interrupts upon the $360 / 95$, (as these negate the advantages of its special hardware, such as that which permits the concurrent execution of instructions) SUPERMON reported that the printers (the $360 / 95$ is equipped with five) were the major source of $I / O$ interrupts, accounting for about one-half of the prime shift rate of approximately 15,500 interrupts per minute. Accordingly, the software routines supporting the printers were examined and were altered. Our studies show that the changes to these routines have reduced the printer I/O interrupt rate by a factor of 16 .

\section{Link Pack Area}

Information from SUPERMON has also resulted in a reconfiguration of the system modules that reside in the system's Link Pack Area (LPA). The presence, in LPA, of frequently used modules that can be shared by concurrently executing programs eliminates the need to load a copy of the routine each time it is required by a program or system task and also reduces that program's or task's memory (i.e., REGION) requirements. SUPERMON identified a group of about 25 modules residing in LPA that averaged only about one use every 2 minutes and also revealed about 40 modules not residing in LPA that averaged a usage rate about 1,200 times greater. So, replacing the first group of modules in LPA with the second group eliminated about 600 loads per minute from system libraries on direct access devices at the small cost of increasing the size of the LPA by $10 \mathrm{~K}$ bytes. (The 360/95 has 5 million bytes of memory.)

The reader should be cautioned, however, that indiscriminate inclusion of modules in the Link Pack Area may actually cause a decrease in system performance. An example of this is the overlay supervisor module, IEWSZOVR, which at times has been used on the 360/95 at a rate of over 630 accesses per minute as measured over a 5-hour period by SUPERMON. While the frequent use of this module would seem to make it a prime candidate for inclusion in LPA, studies showed that by doing so the $\mathrm{I} / \mathrm{O}$ charge of some jobs was increased by as much as a factor of 30 while the $I / O$ charge of the rest remained unaffected.

\section{Memory Utilization}

Early studies done with SUPERMON indicated that the amount of memory on the $360 / 95$ available to process user jobs was becoming critically short. The problem became even more serious after a local modification to the operating system allowed our installation to use more than the "maximum" number (15) of initiators to process the input job stream. In order to monitor this situation, another modification was made to the operating system to print out a console message indicating the region size required by each job and the time spent waiting for memory to become available. As a result of these studies, a concentrated effort started to have the users critically re-examine the REGION requirements for each of their job steps, cataloged procedures were modified to default to reasonable REGION's for compilations, utility steps, etc., and several changes were made to the operating system which caused jobs to fail if excessive (unused) memory demands were made. Assignment of priorities to jobs in the input job queue on the $360 / 95$ according to the maximum CPU or I/O time estimates of a job (required on GSFC job cards) was also made and turned out to be quite satisfactory. However, it appeared that in order to utilize multiprogramming more fully, I/O-bound jobs should have higher priority than CPU-bound jobs during execution. A local modification was made to the operating system which assigned execution (or run time) priorities to jobs according to the ratio of I/O to CPU time required. Thus, at this installation, a distinction is made between job selection (or initiation) priority, and execution (or run time) priority. With the use of SUPERMON and the Boole \& Babbage system monitoring programs, it was found that average CPU utilization increased by over 4 percent as a result of this change.

In order to study the effect on the turnaround time of the job stream, a special report was generated based on the System Management Facility (SMF) accounting data collected for each job. As a result of this study, it was found that turnaround times had decreased quite dramatically, and on the days of the test, it was found that the average turnaround time of all the jobs submitted decreased by almost 10 percent.

Periodically, certain types of SMF records are collected and interpreted with an installation-provided formatting routine, which gives the frequency of usage of all programs executed on the system. As a result of this, optimizations have been made in both the cataloged procedures that call these programs and in the programs themselves (wherever possible) for the most heavilyused programs. In some cases, users have been advised to utilize more efficient programs to carry out the same functions. An example of this is the usage of Assembler $G$ rather than Assembler F, or the use of LISTPDS rather than IEBPTPCH. In both cases, it was found that the users could carry out the same functions as before, only much more efficiently.

\section{Optimization of the Workload}

The foregoing discussion has dealt primarily with efforts to balance the computing facility and optimize the operating system in processing the workload, without regard for the level of efficiency of individual programs. However, it has been recognized that improvements in applications programs would also lead to increased machine performance separate from and in addition to the benefits of system tuning. Considerable attention has been given to this matter, with most gratifying results. There are literally thousands of programs involved, and even after restricting this to consideration of frequently recurring production jobs, the magnitude of the task is quite high. First attention has been given to these programs which are run most frequently, which absorb significant amounts of machine time and/or resources, which most seriously impact the overall operation of the machine, or which are observed to overload the available facilities most heavily.

A number of these programs were subjected to PPE analysis and the time-consuming areas of code thereby isolated. In 
general, these areas of code were reworked to produce identical results but eliminate the inefficiencies. Detailed descriptions of each line of code which were modified would be unwieldy, but in general the following coding practices were found:

a. Large numbers of labeled COMMON areas in FORTRAN programs.

b. Redundant calculations.

c. Complex (factored) mathematical expressions.

d. Large numbers of calls to FORTRAN mathematical library routines.

e. Excessive number of calls to FORTRAN I/O routines.

f. Unblocked or inefficiently blocked data sets.

g. Unnecessary subscript calculations especially in multi-

dimensional arrays or excessively complicated subscript notation.

h. Mixed mode expressions and unnormalized arithmetic.

i. Floating point exponentiation.

j. Excessive use of arithmetic "IF" statements.

k. Excessive use of "DO loops."

1. "Out-of-line" coding of functions.

Substituted Practices:

a. A number of labeled COMMON areas were combined thereby freeing up the base registers assigned to each and effectively reassigning them to computational functions.

b. Redundant calculations were eliminated.

c. Expressions were unfactored to eliminate unnecessary calculations.

d. Calls to FORTRAN library subroutines were minimized either by substituting "in-line" code, assigning previously calculated values to temporary save variables, or using trigonometric identities from previously calculated values.

e. Array reads and writes were substituted for subscripted reads and writes. In cases where the program was writing data to a device for subsequent retrieval and manipulation, unformatted reads and writes were substituted.

f. Where possible, blocking factors for data sets were increased to the maximum capability of the device, thereby minimizing the number of actual I/O operations.

g. Where possible, arrays were reduced to a single dimension eliminating excessive overhead in subscript calculation.

h. Mixed mode expressions were eliminated where possible. Where these expressions could not be fully eliminated, calculations for each mode were grouped. Moreover, arithmetic was redesigned to minimize normalization.

i. Fixed point exponentiation was substituted for the floating point type, and in those instances where variables are multiplied by themselves, fixed point exponentiation was substituted.

j. In all possible instances, logical "IF" statements were substituted for the aritlumetic "IF" variety.

$k$. Loops of clearly defined length were written out, thereby eliminating the index calculation and the overhead associated with the DO loop.

1. Where function subprograms were used, this code was recoded "in-line" eliminating the overhead associated with function references.

Six major programs subjected to PPE analysis during CY71 resulted in a demonstrated yearly cost savings of $\$ 223,696$. Improvements of this type and magnitude can be made only with the sincere cooperation of the originator. In fact, it is generally more appropriate and expedient for the user himself to incorporate and test any changes to be made but with the assistance, guidance, advice, and encouragement of systemoriented personnel.

Of particular interest is a by-product of the Boole \& Babbage activities. Many of the logical construction in the FORTRAN language which have been found to be inefficient are repeated, independently, by many users. Just the process of locating these inefficiencies separately for each job examined is, itself, inefficient. Our Boole \& Babbage representative authored a clocument (S-543-71-99) which delineates good and bad FORTiRAN programming practices. This document which was later up tated and republished as X-530-77-72 has been widely acclaimed as a very useful aid to the programmer, not only at this Center, but at other installations both within the Government and pivate industry as well.

In addition to the intensive software analysis for incre asing computer efficiency, active aid is being provided to the computer users through our Programmer Assistance Center (F'AC) staffed by four systems-oriented personnel. On occasion, a user may recognize that there are inefficiencies in his own prograr: and will seek the advice of suitable personnel. At other times, the opportunity for improvement may be observed accidentally, as when the Programmer Assistance Center is aiding a user and happens to spot an unrelated aspect of the code wherein improvements can be made. Also, situations may arise whe rein a user is forced to seek assistance in order to obtain acceptable service from the machine.

The PAC identified, thus far, some of the difficulties $\mathrm{x} x$ perienced by our users as explained below and provided ef ective solutions to some of these, while offering recommended circumventions to others.

a. Some of the large programming systems at Goddard were developed for the earlier and less sophisticated computers. Present-day computing systems are much more capable and can perform these jobs more effectively. To take maximum advantage of this capability, advanced programming techniques and program design must be considered.

b. Some of the programmers who developed these programs originally are no longer available, and their successors, being unfamiliar with the underlying logic, must guess as to why certain things are done in certain ways.

c. Several of the programs examined show lack of proficiency in the effective use of the Job Control Language (JCL).

The Programmer Assistance Center approaches this problem from several points of view.

First, it examines each job brought to it for possible improvements rather than just to answer a specific problem which may be presented.

Each morning, the PAC polls the computer operators to find out about any jobs that have caused operational problems ind are therefore candidates for improvements. When a job :s selected, it is thoroughly examined, and if any worthwhile : :uggestions can be made, the user is contacted, the problem is explained, and assistance is offered.

Prior to the start of the normal work day, the output in the dispatch office is examined. Jobs requiring high CPU or $I / O$ time are sought out as well as any jobs appearing to demand an excessive amount of resources such as large volumes of paper or cards. The findings of the PAC are passed on to the individual user or user group.

The knowledge gained is shared with the user community through the preparation of Computer Bulletins or Compu:ergrams issued by the Computer Management Branch and classroom lectures given jointly by the PAC and Computer Managernent Branch personnel.

Whenever problems occur which adversely affect a numbier of users, the PAC studies them in detail and develops techniques for their effective handling.

Finally, the operational environment itself is researche.1. 
Private user libraries and procedures are examined and recommendations are made whenever applicable. Work of this sort is somewhat slow due to the extensive research and user interface required, but improvements resulting from these efforts are substantial. Some of these are cited below.

A group of 200 separate but similar jobs, each of which called upon the same two tapes, was optimized by the user following the PAC recommendations. The following gains were achieved:

a. Over 100 hours of tape-drive tie-up was freed by eliminating the need for tapes.

b. Operator intervention previously required by each job was eliminated.

c. Saved over 3 hours of $\mathrm{I} / \mathrm{O}$ time.

d. Saved over 1 hour of 360/95 CPU time.

e. Eliminated over 7,000 pages of printer output.

f. Reduced each job's scratch disk requirements by 60 cylinders ( 1 cylinder $=145,880$ bytes).

g. Reduced by one-half, the job queue space required for each run.

A priority production run which daily uses from 10 to 30

minutes of 360/95 CPU time and from 20 to 45 minutes of $1 / O$ time (depending on the investigation) was examined by the PAC, and a series of recommendations were proposed. Some of these have already been implemented, and the program now uses from 1 to 5 minutes CPU time and 1 to 8 minutes $I / O$ time. The main suggestion was to combine seven separate job steps into one and to produce each of the different types of reports needed in a single pass. As a demonstration, the PAC combined the first two steps into a program that runs in one-third of the CPU time and one-fourth of the I/O time previously required by these two steps. The user has implemented the remainder of the recommendations.

One user group was experiencing a high program failure rate attributed to tape errors. After detailed study, the PAC eliminated the majority of these errors by simple changes to the JCL. Approximately one year later the same group began experiencing additional I/O problems. Further suggestions were made in an 18-page report explaining the nature of the difficulties. Since this group is one of the heaviest users of the $360 / 95$, substantial gains have already been made and are expected to continue. For example:

a. The programs now terminate with a diagnostic dump whenever an I/O error occurs. Previously, such a job often timed out wasting from 20 to 60 minutes of CPU time.

b. An average reduction of over 100 cylinders of disk space required by the programs eliminated most of the failures due to insufficient disk space.

In the area of excessive paper consumption, the PAC has aided several users to reduce their demands on this resource. For example:

a. One run was found and corrected that had an erratic print control that caused excessive printer spewing and paper jamming. Estimated savings of one box of paper every 2 weeks.

b. A production graphics run was optimized wherein the I/O time and paper consumption were halved. Estimated savings of one box of paper per run. This program run occurred about three times per week.

c. One repetitive message that occurred across many different runs of a large user group was eliminated with an anticipated savings of one-half a box of paper per day.

d. One user group was aided in improving their source deck handling procedure with an immediate savings of 9,000 punched cards with further savings expected as additional deck requests are eliminated.
Fifty-four 10-minute runs were optimized producing the following gains:

a. Need for tape was eliminated.

b. Need for operator intervention was eliminated.

c. Memory tie-up for wait on device allocation was eliminated.

d. One-half hour of $1 / O$ time was saved.

e. Ten minutes of CPU time was saved.

A series of 22 jobs which used the same tape and required operator intervention were optimized eliminating the need for tape and operator intervention.

A regular production job which required four tape drives to execute was changed to require only two.

One user group was found to be writing unblocked records to tape. Upon the PAC's recommendation, the records were given a large block size and recorded at a higher density with the result that for a full reel of tape:

a. The $\mathrm{I} / \mathrm{O}$ time dropped from 21.6 minutes to 1.5 minutes.

b. The capacity increased from 40,000 records per reel to 560,000 .

Telemetry data being gathered onto a tape from the $360 / 95$ was being stored in an improper format. PAC recommendations reduced $\mathrm{I} / \mathrm{O}$ time needed to run from 25 minutes to 2 minutes for a daily production run.

\section{Optimization of the New Workload}

The third item of management policy directed toward improving the productive use of these computers is intended to assure that new applications programs are written in an optimum fashion. This is achieved through training programs designed to educate computer users in the proper use of the equipment and software employed on M\&DO S/360 equipment. Historically, changes in computer technology, such as languages, compilers, operating systems, documentation aids, etc., have brought changes in programming methodology. Third generation software is far more complex than most people realize. This complexity, however, can be an advantage if adequate emphasis is placed on training. Through training, programmers learn how to take full advantage of the theoretical possibilities offered by the system, how to comprehend all the complex factors within a multiprogramming environment which affect organization and selection of software, performance of hardware (especially as influenced by applications program design), and how to design programs which operate efficiently once in production. Formal training classes are being conducted on Time Sharing Option (TSO), Job Control Language (JCL), Overlay Structures, and MVT Dump Interpretation. In addition, self-teaching courses on audio-video tapes which the users may receive include Data Processing Concepts which describes the computer, its design, functions, devices, and operations; Fundamentals of Programming dealing with problem analysis, program loops, and table operations; ANS COBOL and COBOL Programming Efficiencies; Introduction to $S / 360$ which introduces the student into instruction formats, numbering systems, programming support, and multiprogramming; OS System Service Programs and OS Data Management which provide an understanding into utilities and their control, combining modules and modular programming, SAVE and RETURN, OPEN and CLOSE, and QSAM and BSAM examples; Assembler Language Coding which is an introduction to programming languages, load and branching instructions; Computer Operator Training in I/O Devices; Data Base Concepts and Methods which include data management techniques, file organization data base, queries, case study, and solutions; Teleprocessing Systems which describe communication engineering, terminals, network design, channels, and aid programs; 
Asymmetric Multiprocessing System (ASP) which is a description of ASP initialization and floor demonstration of the operation of ASP.

The results of our training efforts have been evident in the continual increase in computer throughput, substantial decreases in individual programmer difficulties, and the creation of large, complex applications systems capable of meshing smoothly with OS/360 and with each other in a multiprogramming environment.

\section{Communication with the Users}

It is a well-known fact that communications exchange must be both continuous and reciprocal. Good communications do not just happen. One must do a lot of work to make them happen. Toward this end a computer users committee was formed, which meets once a month on a regular basis and more often when necessary.

Gatherings of this type serve several purposes. They link the users together for the purpose of collectively suggesting improvements to the overall computer services provided. They also serve as a platform for management to translate organizational objectives and policies in terms that the users can understand and accept. In addition the Computer Newsletter, the Computergram, and Writer News are publications designed to make known in advance, forthcoming policies, changes in operational procedures, acquisition, deletion, or modification of hardware or software and their impact on the users. They also provide tips and suggestions on how to avoid common or expensive mistakes.

Although the examples cited above are limited to the productivity improvements made through 1974 , it should be obvious to the reader that continuous improvements are being made daily. Furthermore, benefits derived from these improvements are carried through the years. In spite of the fact that computer resources were dedicated for special purposes and the job stream has increased in both magnitude and interactive complexity, the productivity of the $360 / 95$ has increased from about 400 jobs daily to over 1500 jobs today. Savings of millions of dollars were also made through eliminating the need for additional hardware acquisition in order to meet the increased demands on the facility.
The degree of success attained through this management approach is amply proven by the fact that the General Azcounting Office (GAO), Office of Management and Bud get (OMB), NASA Headquarters, as well as other NASA Centers and Agencies, look upon this Center with admiration and respect. There are several examples which may be cited to substant ate this claim. GAO investigated the Automatic Data Process.ng (ADP) activities of this Center quite thoroughly. Their resultant reports to the Congress of the U.S.A. dated August 22, 1972, and June 3,1974, were quite favorable and complimentary to the GSFC. For example in their June 3, 1974 report, GAO states:

"A group at the Goddard Space Flight Center, whose only tool was its knowledge of efficient programming techniques, was able to save about $\$ 300,000$ annually, encompassing CPU, input-ou:tput, programmer, and operator time, and magretic tape, paper, and cards. An average of 300,000 locations in internal storage have been made available for multi-prograr uming and $15,000,000$ locations in external storage have been made available to other programs.

Another group, which used a software monitor, saved the Center over $\$ 750,000$ of computer time in 1 year by increissing the efficiency of key application programs."

These two groups are the Programmer Assistance Center and the Boole and Babbage Office, both managed by the Mission Operations Computing Division. Eliciting this sort of response from GAO, which is necessarily a critical agency, is most unusual. On another occasion, a group of individuals from NASA Hieadquarters and the Kennedy Space Center selected the Computer Management Branch as a source of guidance in the use of performance measurement techniques. This contact, which resulted in a meeting held on May 1, 1972, indicates the recognition accorded us as being exceptionally knowledgeable in this field. On August 18, 1971, a task force led by OMB visited GSFC' as part of a study directed at how various Government agencies were managing their computing facilities. Apparently, this 'Denter impressed them favorably since the same individual from $O M B$ returned on May 18, 1972, along with two colleagues, to discuss with MOCD personnel software and facility management aspects of setting up their own computer facility, a proposed $370 / .55$. 